\title{
Vaccines beyond 2000
}

Measles could be eradicated on a global scale by the year 2007, according to Sir Gustav Nossal, president of the Australian Academy of Science and former head of the Walter and Eliza Hall Institute of Medical Research in Melbourne. Nossal also forecasts that new vaccines against cholera, pneumonia, respiratory disease, gastritis, diarrhea and meningitis will become widely available by the turn of the century.

Nossal made his predictions at the recent "Vaccines Beyond 2000" international conference on Australia's Gold Coast, and told delegates that they should aim higher than merely controlling infectious disease. "Eradication is a wonderful prize," he said.

As chairman of the World Health Organization's (WHO) Scientific Advisory Group of Experts (SAGE) to the Global Programme for Vaccines and Immunisation (GPV), Nossal is better placed than most to assess the worldwide status of infectious diseases. The GPV not only provides information and guidance concerning aspects of vaccine supply, quality, immunization and disease control, but also supports the financial development of new vaccines.

Although many question

Nossal's optimism and say it will take at least a decade before global use of many of the newer vaccines becomes a reality, his enthusiasm is understandable in light of the WHO's highly successful polio vaccination program. Polio cases world-wide have fallen by more than $90 \%$, and the disease is likely to be eradicated by the year 2000 , thus meeting the target set by the World Health Association in 1988, and saving governments around $\$ 1.5$ billion each year in immunization costs.

Unfortunately, the fight against other diseases has been less remarkable. Twelve million children under five still die from infections each year and two million of these fatalities could be prevented with existing vaccines. In 1994, more than a million children died from measles, close to 500,000 from neonatal tetanus and almost 400,000 from whooping cough, the WHO reports.

Mass immunizations have been hampered by political and financial constraints, as well as what some call "donor fatigue." In recent years, most developed countries (donors) have reduced significantly their international aid budgets. One casualty of these changes is the hepatitis-B vaccine, which has been in existence since the 1980s, but has still not been distributed to third world countries where it is needed most. infected with the bacterium and vaccination is the only practical solution for large-scale prophylaxis, he has received little support from WHO. Douglas Lowrie of the National Medical Research Council of the UK, also complained of receiving little WHO support for the development of a genetically engineered vaccine against tuberculosis.

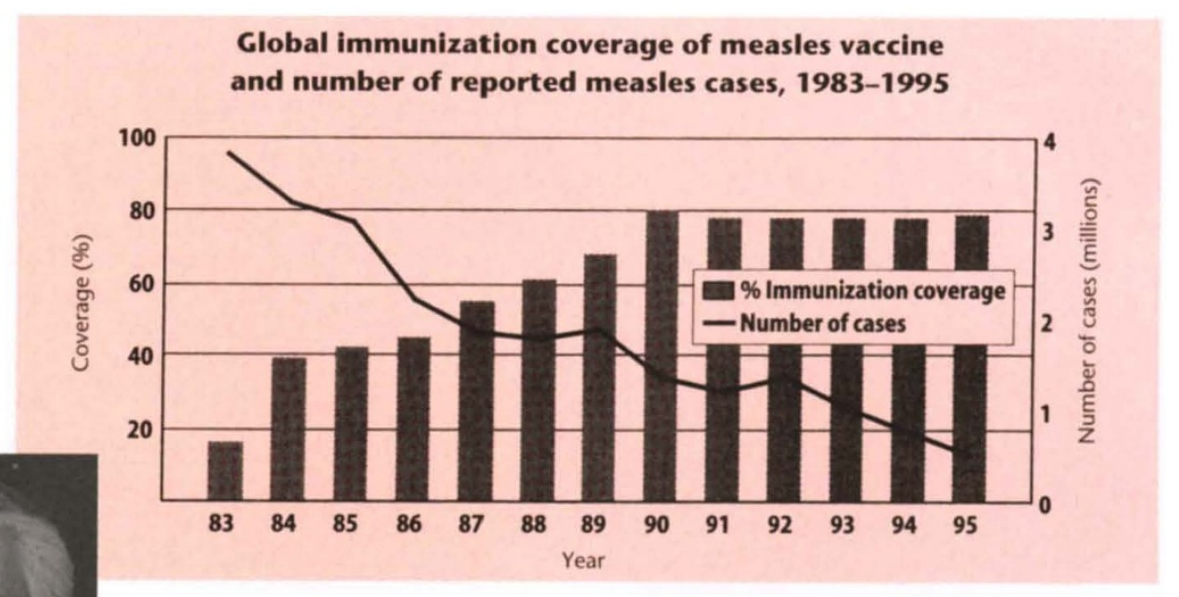

And even at a cost of $\$ 0.17$ per patient, many African countries have been unable to afford much needed vaccines against yellow fever.

As the cost of research in general - and developing new vaccines in particular - escalates, $\mathrm{R} \& \mathrm{D}$ investment has also been reduced for many diseases.

Conference delegate Adrian Lee, who leads the development of a vaccine against the gastritis-causing bacterium, Helicobacter pylori, at the University of New South Wales, says that although more than half the world's population is
Despite these rumblings, others acknowledged WHO support, and there was a general consensus at the meeting that the WHO is doing a good job, although new sources of financing will have to be developed to improve its efforts.

Nossal agrees that new forms of financing must be put in place to ensure that novel vaccines are widely used as soon as they reach the marketplace. He cited the World Bank as the best placed institution to get intimately engaged in financing GPV initiatives. These discussions are currently under way.

ELIZABETH BAN Sydney

\section{Tackling the scourge of river blindness}

The World Health Organization (WHO) and the World Bank together with 11 other organizations, including the Carter Center and Merck and Co. (the only commercial venture in the group), recently declared an ambitious initiative to eliminate river blindness (onchocerciasis) from Africa by 2007 . Caused by the worm Onchocerca volvulus, this disease is the second leading cause of blindness in the developing world and number six on WHO's list of most devastating tropical diseases. The initiative will expand existing programs, which use community-based treatment with the drug ivermectin and vector control, into all regions of Africa.
Merck announced its commitment to produce and donate sufficient ivermectin to meet the initiative's goal. The declaration auspiciously coincides with the tenth anniversary of Merck's announcement to distribute ivermectin $\left(\right.$ Mectizan $\left.^{\mathrm{tu}}\right)$ - calculated to cost $\$ 3$ a tablet - free of charge to any country with endemic river blindness. "This declaration is important because it affirms in writing the world's intent to help Africa get rid of river blindness," says Donald Hopkins, Associate Director for Health Programs at the Carter Center in Atlanta, Georgia, which coordinates distribution of the drug.

ORLA SMITH 